\title{
QUANTUM GRAVITY
}

\section{FROM THE ENTROPY OF GEOMETRIES}

J. Ambjørn ${ }^{1,3}$, A.T. Görlich², J. Jurkiewicz ${ }^{2}$, R. Loll ${ }^{3}$ - DOI: 10.1051/epn/2011204

The Niels Bohr Institute, Copenhagen University, Blegdamsvej 17, 2100 Copenhagen Ø, Denmark - ambjorn@nbi.dk

nark Kac Complex Systems Research Centre, Marian Smoluchowski Institute of Physics, Jagiellonian University, Reymonta 4, 30-059 Krakow, Poland atg@th.if.uj.edu.pl - jerzy.jurkiewicz@uj.edu.pl

Institute for Theoretical Physics, Utrecht University, Leuvenlaan 4, NL-3584 CE Utrecht, The Netherlands - r.loll@uu.nl

This article describes an attempt to reconcile the theory of general relativity with quantum theory from first principles. The universe is assembled from building blocks, and computer simulations reveal their collective behaviour. For small universes quantum fluctuations are large and dominant, but still some semiclassical concepts of geometry survive.

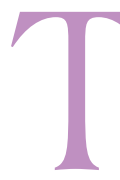

hree constants play a fundamental role in our understanding of nature: the gravitational constant $G$, which quantifies the strength of the gravitational forces in Newton's law, the velocity of light $c$ appearing in Einstein's theory of Special Relativity, and Planck's constant $\hbar$, which he first introduced to describe the thermal spectrum of blackbody radiation. These three constants are associated with different aspects of our universe.

Newton's law of gravity governs the motion of the solar system, which means that $G$ is primarily associated with physics on large scales. The theory of Special Relativity determines physics at extreme velocities close to the velocity $c$ of light. Finally, Planck's constant $\hbar$ appears in a central way in Quantum Mechanics, the laws of physics prevailing in the micro-cosmos.

Einstein's theory of General Relativity unites the physics defined by the constants $G$ and $c$. It describes how energy and matter deform ("curve") the geometry of space-time. As far as we can tell from astrophysical observations, the theory also provides us with a good description of the dynamics of our universe as a whole. On the other hand, the theories of Special Relativity and Quantum Mechanics, defined in terms of $c$ and $\hbar$, are merged into the 
- common theoretical framework of Quantum Field Theory. While there are many quantum field theories, the so-called Standard Model has been selected experimentally. It describes the atomic and subatomic world down to a scale of $10^{-18} \mathrm{~m}$ with amazing precision.

\section{A single theory?}

In view of the above description it appears natural to try and take the "final" step of unifying General Relativity and Quantum Field Theory into a single theory (Fig. 1 ), with all three coupling constants, $G, c$ and $\hbar$ expected to play a role.

A natural unit of length in the combined theory can be obtained by multiplying appropriate powers of these three coupling constants. The resulting Planck length is unbelievably small, a mere $1.6 \times 10^{-35} \mathrm{~m}$. This should be compared with the length scale we can presently probe directly in particle accelerators, which is around $10^{-18} \mathrm{~m}$ It leads us to conclude that Quantum Gravity, the soughtfor theory unifying Quantum Field Theory and General Relativity, will most likely manifest itself at or near the Planck scale, which unfortunately lies far beyond the reach of any direct verification in the laboratory.

The lack of guidance from experimental or observational evidence has led researchers to consider a number of candidate theories, of which String Theory is probably the best known. While these theories provide at least partial descriptions for what may go on at the Planck scale, they have difficulties in explaining the fourdimensional world as we know it at macroscopic distances. This is a somewhat paradoxical situation since exactly at those scales we have access to experimental data.

There is an interesting precedent for this kind of situation in the theory of Strong Interactions. It describes the forces binding an atomic nucleus together, and how the proton and neutron are composed of three quarks interacting by the exchange of gluons. It is an example of a quantum theory whose short-distance, "microscopic" physics we actually understand. Although it should in principle also give an accurate description of "macroscopic" physics - by which in this context we mean the properties of the proton, the neutron and other observed hadrons - one is presently unable to perform the analytic calculations to prove it. In this situation, the computer comes to our help: we can represent the world as a lattice (with as small a lattice spacing as possible) and run computer simulations of the lattice theory. In this way we can determine the mass ratios of neutron and pion or of proton and pion, say, and check that they agree with experiments.

\section{Amazing simplicity}

Is it possible to do the same with our universe? Can we take our favorite, microscopic theory of Quantum Gravity, squeeze the whole universe into the computer and establish whether the macroscopic part of the theory gives rise to anything resembling our present universe? Surprisingly, the answer is in the affirmative, at least for the specific theory that goes by the name of "Causal Dynamical Triangulations" or "CDT" for short. One virtue of this Quantum Gravity Theory is its amazing

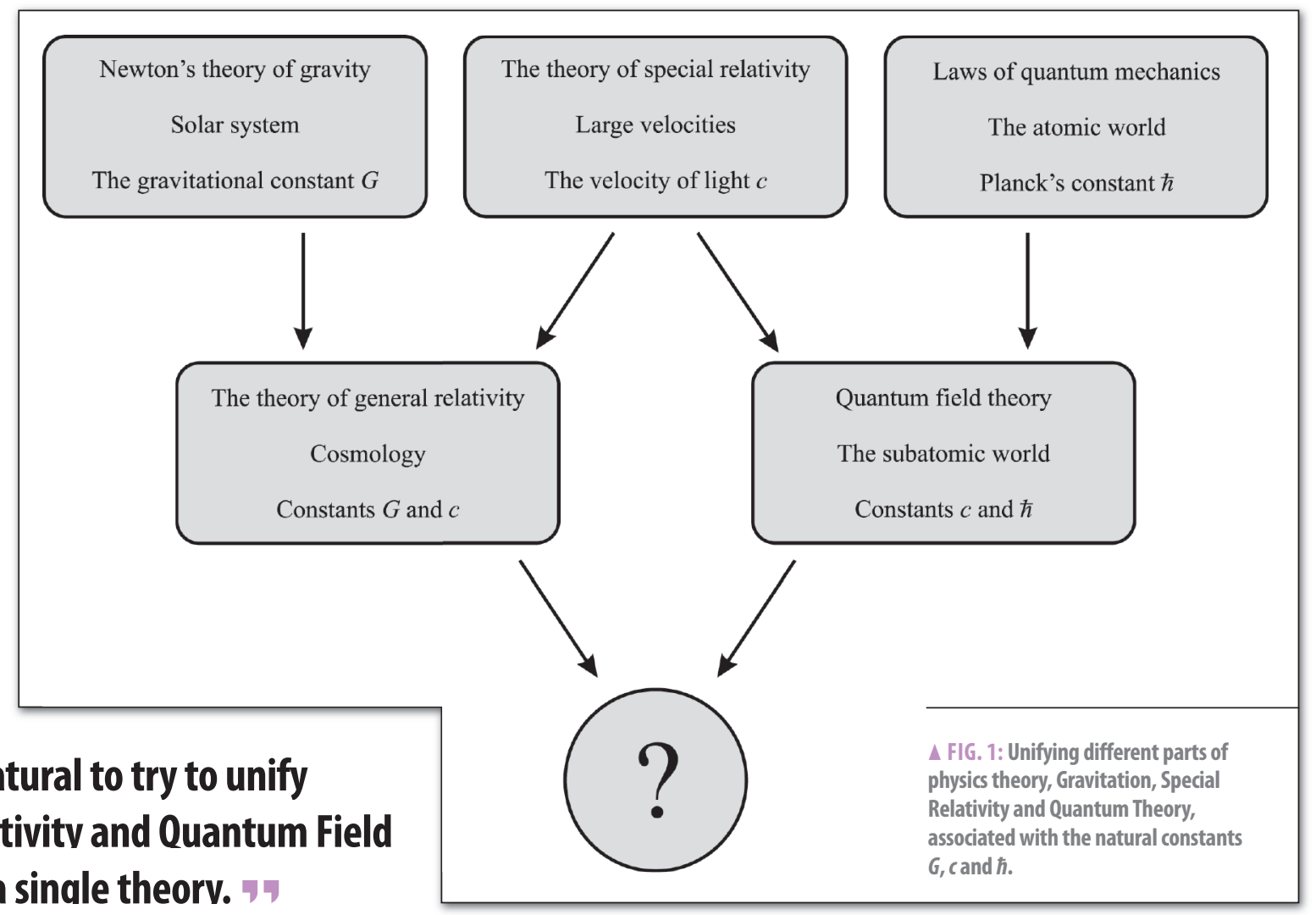


simplicity. In it, space-time is assembled from tiny, elementary building blocks in the form of "four-simplices", four-dimensional generalizations of triangles with one time and three space dimensions. To obtain a Quantum Theory of Gravity one then performs a summation (a "quantum superposition") of all possible space-times with appropriate weights, following general principles from Quantum Field Theory.

The theory could be solved analytically if one could count the number of ways of constructing different universes by gluing together four-simplices, in other words, the number of microstates the system can occupy. This state counting or entropy is decisive in determining the quantum dynamics of the system. Since we are unable to do the counting analytically, we let the computer take care of the task. A typical simulation uses a universe size of 40.000 to 360.000 four-simplices. It is important to appreciate that we do not consider these triangular "building blocks" as a fundamental substructure of space-time, but merely as a convenient tool for modeling its microscopic degrees of freedom. We are only interested in space-time properties that persist when the block size is shrunk to zero. This is analogous to modeling a large-scale shape, like Einstein's face shown in Fig. 2, by Lego blocks. Obviously, we will get a better and better approximation of the "real" Einstein by using ever smaller Lego blocks.

In our case, the "lattice" is therefore a four-dimensional triangulation of space-time.

\section{Quantum fluctuations}

However, unlike the lattices used in computer simulations of the Strong Interactions, this lattice is dynamical: the computer changes it all the time, reflecting the fact that in a theory of Quantum Gravity space-time itself constantly undergoes quantum fluctuations. We expect to observe an "average" macroscopic universe, where the deviations of any given computer-generated configuration from this average will constitute the quantum fluctuations. Fig. 3 shows a two-dimensional projection of such a universe as it appears in the computer.

Had we randomly selected another configuration, it would look somewhat similar but not identical. By superimposing many such configurations an "average" configuration will emerge. The beautiful surprise is that (within computing accuracy) this average configuration is a solution to the classical Einstein equations of Gravity. One may wonder why the fluctuations around the average are rather large, whereas we do not observe anything of the kind in our real universe. The point is that the (relative) magnitude of the quantum fluctuations depends on the size of the universe - the smaller the universe, the larger the fluctuations - and that our computer-generated space-times are rather small. Our computer simulations are currently limited to about 300,000 four-simplices. If one translates this into the

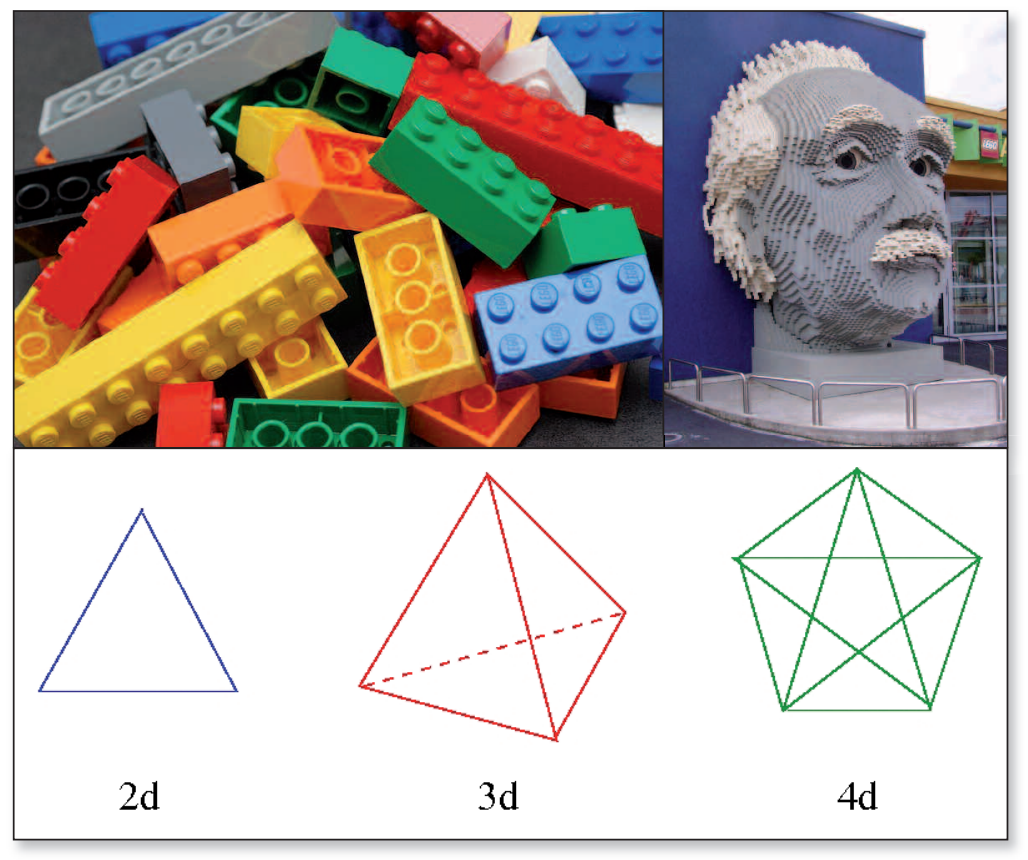

$\Delta$ FIG. 2: Building blocks used for Einstein (Lego blocks) and, analogously, building blocks for curved geometries in dimension 2 (triangle, with one-dimensional surface formed by 3 edges), dimension 3 (tetrahedron, with two-dimensional surface formed by 4 triangles), and dimension 4 (four-simplex, with three-dimensional surface formed by 5 tetrahedra).

more familiar setting of a four-dimensional, regular (hyper-)cubic lattice, the triangular building blocks would approximately fill a lattice of dimensions $10^{4}$. In fact, it is quite remarkable and encouraging that "on average" we can observe a good classical behaviour for triangulations with relatively few four-simplices.

We have not yet revealed which solution to General Relativity emerges from our quantum construction. If in Fig. 3 we follow the development of spatial slices in time by moving upwards, we see that the universe initially has no spatial extension, after which it expands to some maximal volume, and then recontracts to a point, continuing with no spatial extension. After averaging over many such configurations, the geometry of a spatial slice within the "blob" turns out to be that of a three-dimensional sphere, whose radius changes in time such that the total blob is a perfect, round fourdimensional sphere. This one recognizes as a solution to the classical Einstein equations with a positive cosmological constant in so-called Euclidean space-time.

In Euclidean space-time, "time" is treated as just another spatial direction, rather than a time direction. One of the nice features of Causal Dynamical Triangulations is that to each "real" geometry with a distinct time direction one can associate a corresponding Euclidean geometry, where the time direction has been replaced by a space direction. Just as in testing the theory of strong interactions by computer simulations, also here it is convenient from a technical point of view to carry out the calculation using a four-dimensional space, rather than a true space-time. 


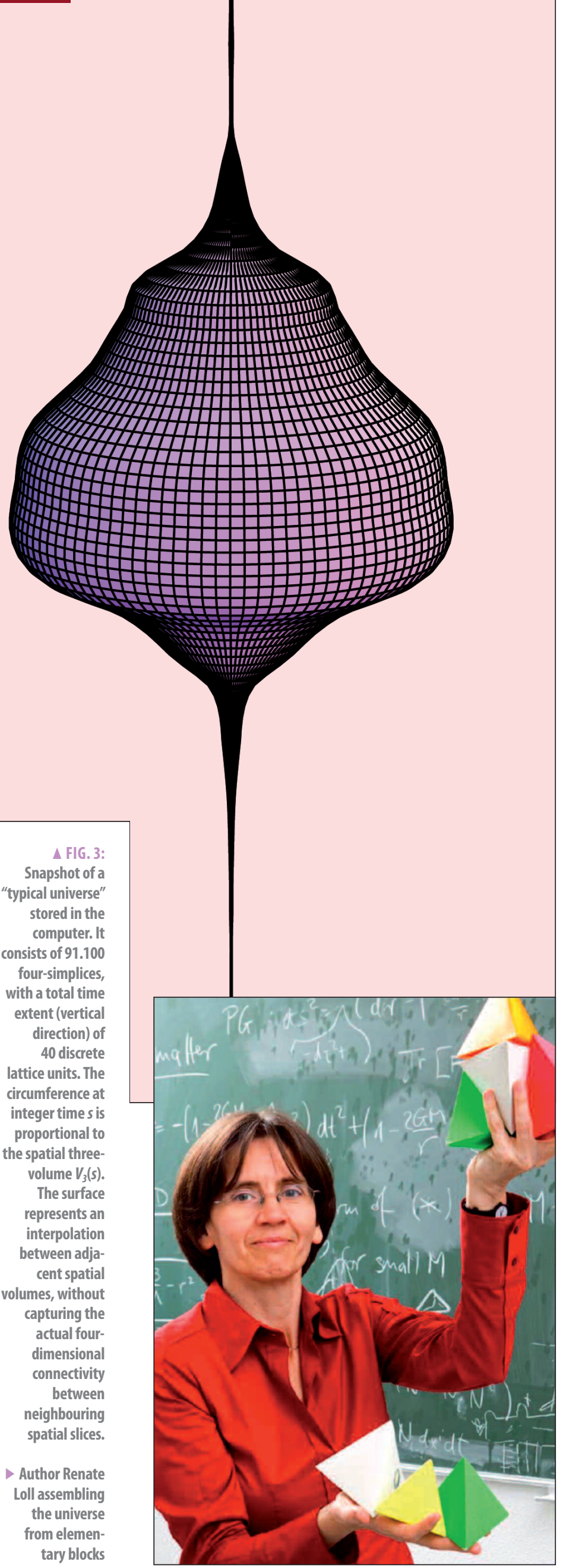

\section{Expandling universe}

When we say that a classical universe emerges from the computer simulations, it is in this Euclidean sense. Upon rotating this solution back to "real" time, we obtain a real, physical solution of classical Gravity known as de Sitter space-time. This is a universe which expands exponentially fast, with expansion rate dictated by the cosmological constant. It does not describe our present universe accurately, because we have not yet included matter and radiation in our theory of Quantum Gravity. However, it matches perfectly with current astrophysical observations for the future of our universe, which point to an ever accelerating state of expansion, governed by a cosmological constant, and resembling more and more that of a pure de Sitter space-time.

Can CDT Quantum Gravity also give us insights into the truly nonclassical, quantum features of space-time on short scales? To address this issue we have studied a diffusion process on our ensemble of space-time geometries. One can study diffusion on very general spaces, as long as some notion of distance is present, in order to learn about their geometric properties. The manner and speed of a diffusion process can provide information about the fractal structure of the ambient space. More specifically, it allows one to extract a "dimension" of the space, called its spectral dimension. For any smooth, classical space-time this will agree with its ordinary dimension. For more general spaces, for example, fractal ones, the spectral dimension may assume a whole range of (typically non-integer) values. Intriguingly, while CDT's quantum space-time at large distances appears to be four-dimensional with regard to diffusion (as well as from any other point of view), when we go to small distances it appears to have spectral dimension two. This is a distinct quantum feature of space-time, which has since been corroborated in a different approach to Quantum Gravity, based on a renormalization group analysis. The approach is similar in spirit to ours: one assumes the existence of a Quantum Field Theory of Gravity and then applies general quantum field-theoretic techniques to explore possible consequences. The actual techniques used are very different from ours, so it is encouraging that one obtains the same result.

\section{Literature}

The Self-Organizing Quantum Universe, J. Ambjorn, (Copenhagen U. \& Utrecht U.) , J. Jurkiewicz, (Jagiellonian U.) , R. Loll, (Utrecht U.), 2008; 8pp., Published in Sci. Am. 299N1: 42-49, 2008.

The Self-Organized de Sitter Universe, J. Ambjorn, (Copenhagen U. \& Utrecht U.) , J. Jurkiewicz, (Jagiellonian U.) , R. Loll, (Utrecht U.), ITP-UU-08-34, Jun 2008; 7pp., Published in Int. J. Mod. Phys. D 17: 2515-2520, 2009. e-Print: arXiv:0806.0397 [gr-qc] 